\title{
TANGENTIAL TRAPEZOID CENTRAL CONFIGURATIONS
}

\author{
JAUME LLIBRE ${ }^{1}$ AND PENGFEI YUAN ${ }^{2}$
}

\begin{abstract}
A tangential trapezoid, also called a circumscribed trapezoid, is a trapezoid whose four sides are all tangent to a circle within the trapezoid: the in-circle or inscribed circle. In this paper we classify all planar four-body central configurations, where the four bodies are at the vertices of a tangential trapezoid.
\end{abstract}

\section{INTRODUCTION AND STATEMENT OF THE RESUltS}

The classical $n$-body problem concerns the study of the dynamics of $n$ particles interacting among themselves by their mutual attraction according to Newtonian gravity.

Let $x_{i} \in \mathbb{R}^{d}(i=1, \ldots, n)$ denotes the position vector of the $i$-body, $m_{i} \in \mathbb{R}^{+}(i=$ $1, \ldots, n)$ denotes the mass of the $i$-body. $\mathbb{R}^{d}$ is the Euclidean space $(d=2$ or 3 ). By Newton's law of motion and Newton's gravitational law the equations of the motion of the $n$-body problem are governed by

$$
\ddot{x}_{i}=-\sum_{j=1, j \neq i}^{n} \frac{m_{j}\left(x_{i}-x_{j}\right)}{r_{i j}^{3}}, \quad 1 \leq i \leq n .
$$

Where $r_{i j}=\left|x_{i}-x_{j}\right|$ is the mutual Euclidean distance between the $i$-body and the $j$-body. Here we take the gravitational constant $G=1$.

The vector $x=\left(x_{1}, \ldots, x_{n}\right) \in\left(\mathbb{R}^{d}\right)^{n}$ is called the configuration of the system. Define $\delta(x)$, the dimension of a configuration $x$, i.e. the dimension of the smallest affine space of $\mathbb{R}^{d}$ containing all of the points $x_{i}$. Configurations with $\delta(x)=1,2,3$ are called collinear, planar, spacial, respectively.

When $n=2$ the $n$-body problem has been completely solved. However for the $n$-body problem for $n \geq 3$ the complete solution remains open.

Let

$$
M=m_{1}+\cdots+m_{n}, \quad c=\frac{m_{1} x_{1}+\cdots+m_{n} x_{n}}{M},
$$

be the total mass and the center of masses of the $n$ bodies, respectively.

A configuration $x$ is called a central configuration if the acceleration vectors of the $n$ bodies are proportional to their positions with respect to the center of masses

2010 Mathematics Subject Classification. 70F07,70F15.

Key words and phrases. Convex central configuration, four-body problem, tangential trapezoid. 
with the same constant of proportionality, i.e.

$$
\sum_{j=1, j \neq i}^{n} \frac{m_{j}\left(x_{j}-x_{i}\right)}{r_{i j}^{3}}=\lambda\left(x_{i}-c\right), \quad 1 \leq i \leq n
$$

where $\lambda$ is the constant of proportionality.

A central configuration is invariant under isometries and homotheties with respect to the center of masses. So we say that two central configurations $x=$ $\left(x_{1}, \ldots, x_{n}\right), \bar{x}=\left(\bar{x}_{1}, \ldots, \bar{x}_{n}\right)$ are equivalent if we can pass from one to the other through a dilation or a rotation with respect to the center of the mass. This relation is of equivalence. Therefore when studying central configurations, we only count the classes of central configurations defined by the above equivalence relation.

Central configurations play an important role in celestial mechanics. First, the knowledge of central configurations allows us to obtain homographic solutions of the $n$-body problem (see [35]). We recall that a homographic solution of the $n$-body problem is a solution such that the configuration remains constant up to rotation and scaling. Second, there is a relation between central configurations and the bifurcations of the hypersurfaces of constant energy $h$ and angular momentum $c$ (see $[37,46]$ ). Third, if the $n$ bodies are going to a simultaneous collision or to a total parabolic escape to infinity, then the configuration of $n$ bodies is asymptotic to a central configuration(see $[20,25,43,47]$ ).

There is an extensive literature on the study of central configurations, see Euler [21], Lagrange [28], Albouy [1, 2], Albouy and Chenciner [3], Albouy and Fu [4], Albouy and Kaloshin [6], Davis et al. [18], Hampton and Moeckel [26], Moeckel [36], Llibre [22, 23, 30], Long [33], Érdi and Czirják [20], Moulton [38], Palmore [39], Schmidt [44], Smale [46], Xia [48, 49], Xie [50], ...

In this paper we are interested in the planar 4- body problem. Simó [45] studied numerically the class of central configurations for the 4-body problem with arbitrary masses . In 2006 the finiteness of central configurations for the 4-body problem has been proved by Hampton and Moeckel [26] with the assistance of a computer. Later on Albouy and Kalsoshin [6] proved this result without using the computer.

For $m_{1}=m_{2}=m_{3}=m_{4}$ Llibre found all the planar central configurations by studying the intersections of two curves and assuming that the central configurations have a straight line of symmetry, see [30]. Later on Albouy proved the existence of such symmetry and provide a more analytical proof for the central configurations with equal masses.

When one of the 4 masses is sufficiently small, Pedersen [40], Barros and Leandro $[12,13]$ found the classes of central configurations of the 4 body, also see Arenstorf [11], Fernandes et al [23] and Gannaway [24].

The central configurations with three equal masses was studied by Bernat at al. They classified the non-collinear kite central configurations. For more details, see [14], also see [29].

In 2010 Piña and Lonngi [42] found new properties of the symmetric and nonsymmetric central configurations for the 4-body problem. 


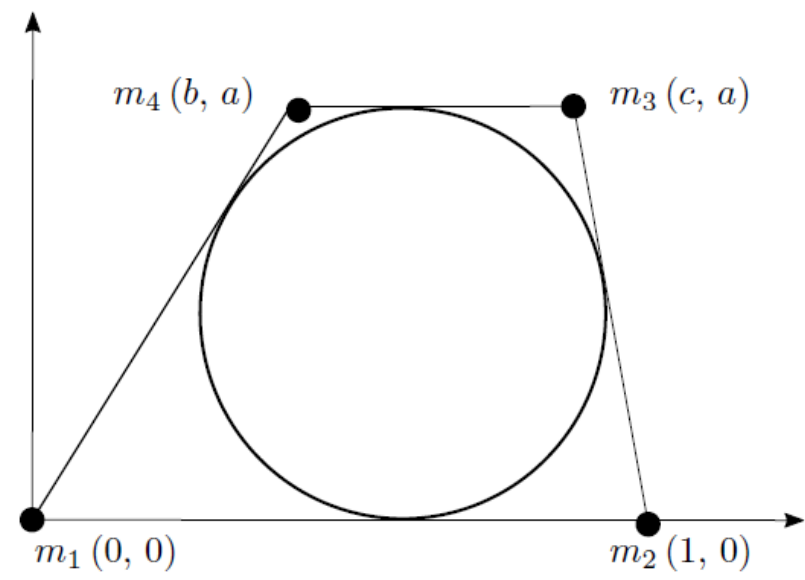

Figure 1. A tangential trapezoid.

MacMillan and Bartky [34] proved that there is a unique isosceles trapezoid central configuration for the 4-body problem when two pairs of equal masses are located at the adjacent vertices of a trapezoid. Long and Sun [33] studied the convex central configurations with three equal masses and they proved that the central configurations must posses a symmetry. Pérez-Chavela and Santoprete [41] generalized further and proved that central configurations must posses such symmetry when two equal masses are located at the opposite vertices of a quadrilateral and at most only one of the remaining masses is larger than the equal masses. Later on Albouy [5] obtain the symmetry of convex central configuration with two equal masses at the opposite vertices.

For $m_{1}=m_{2} \neq m_{3}=m_{4}$ Álvarez and Llibre [7] characterized the convex and concave central configurations with an axis of symmetry.

Using these previous results on the symmetries Corbera and Llibre [15] gave a complete description of the families of central configurations with two pairs of equal masses and two equal masses sufficiently small.

Cors and Robert [16] studied the case when 4 masses are located at the vertices of a cyclic quadrilateral, see also [10].

Recently Álvarez and Llibre [8, 9] classified the Hjelmslev and the equilic quadrilateral central configurations.

The trapezoid central configurations have been studied in [16], here we want to see which of these trapezoid central configurations are tangential.

A tangential trapezoid, also called a circumscribed trapezoid, is a trapezoid whose four sides are all tangent to a circle within the trapezoid: the in-circle or inscribed circle.

Without loss of generality we take $m_{1}=1$ and assume that the positions of four bodies at the vertices of a trapezoid are

$$
x_{1}=(0,0), \quad x_{2}=(1,0), \quad x_{3}=(c, a), \quad x_{4}=(b, a),
$$




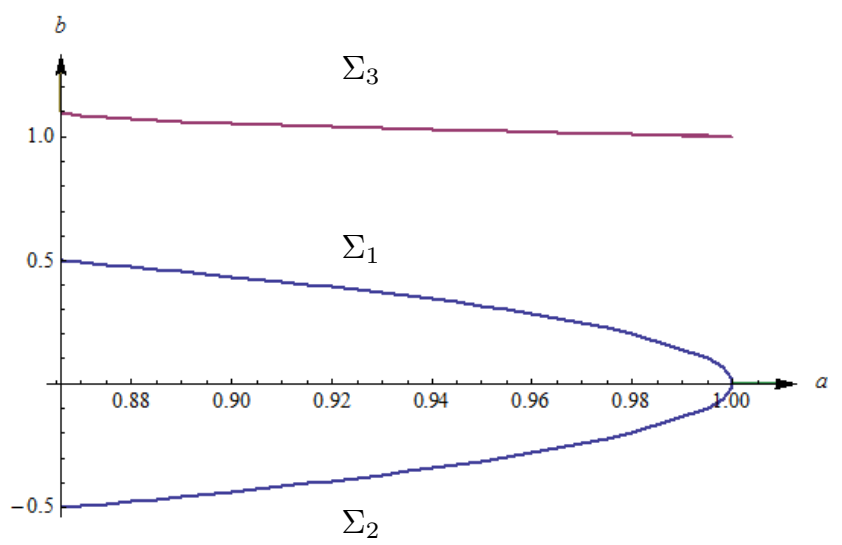

Figure 2. Tangential trapezoid central configurations $\Sigma_{1}, \Sigma_{2}, \Sigma_{3}$.

where $a>0$.

Lemma 1. If the configuration of 4 masses is a tangential trapezoid with the vertices $x_{1}, x_{2}, x_{3}$ and $x_{4}$, as it is shown in Figure 1 , then

$$
c=\frac{(1-b)\left(a^{2}+2\left(b+\sqrt{a^{2}+b^{2}}\right)\right)}{4-a^{2}-4 b} .
$$

Lemma 1 is proved in section 3 .

We characterize all planar 4-body problem central configurations, where the four bodies are at the vertices of a tangential trapezoid.

Theorem 2. We take positive masses for the 4-body problem with $m_{1}=1$. Then we have a tangential trapezoid central configuration given by (2) for each value of $(a, b)$ in the arc $\Sigma_{1}, \Sigma_{2}, \Sigma_{3}$, see Figure 2.

(a) The arc $\Sigma_{1}$ and $\Sigma_{2}$ is symmetric with respect to the a-axis. The arc $\Sigma_{1}$ goes from the point $(1,0)$ to the point $\left(\frac{\sqrt{3}}{2}, \frac{1}{2}\right)$, it is open at $\left(\frac{\sqrt{3}}{2}, \frac{1}{2}\right)$ and closed at $(1,0)$. On $\Sigma_{1}$ we have $m_{1}=m_{3}=1, m_{2}=m_{4}>1$, and $m_{2}=m_{4}$ increases from 1 to $\infty$. The arc $\Sigma_{2}$ goes from the point $(1,0)$ to the point $\left(\frac{\sqrt{3}}{2},-\frac{1}{2}\right)$, it is open at $\left(\frac{\sqrt{3}}{2},-\frac{1}{2}\right)$ and closed at $(1,0)$. On $\Sigma_{2}$ we have $m_{1}=m_{3}=1, m_{2}=m_{4}<1$, and $m_{2}=m_{4}$ decreases from 1 to 0 .

(b) The value $(a, b)=(1,0)$ corresponds to the tangential trapezoid given by the square with four equal masses at its vertices.

(c) The arc $\Sigma_{3}$ goes from the point $(1,1)$ to the point $\left(\frac{\sqrt{3}}{2}, \frac{11}{10}\right)$, it is closed at $(1,1)$ and open at $\left(\frac{\sqrt{3}}{2}, \frac{11}{10}\right)$. On $\Sigma_{3}$ we have $m_{2}>1>m_{3}>m_{4}$, and $m_{2}$ increases from 1 to $1.24789 . ., m_{3}$ decreases from 1 to 0.29184 .., $m_{4}$ decreases from 1 to 0.

(d) The value $(a, b)=(1,1) \in \Sigma_{3}$ corresponds to the tangential trapezoid given by the square with four equal masses at its vertices. 
Theorem 2 is proved in section 3 .

\section{DZIOBEK's EQUATIONS}

In this section we present the equations of the central configurations provided by Dziobek for the 4-body problem.

Let $x=\left(x_{1}, x_{2}, x_{3}, x_{4}\right) \in\left(\mathbb{R}^{2}\right)^{4}$. We associated with $x$ the matrix:

$$
X=\left(\begin{array}{cccc}
1 & 1 & 1 & 1 \\
x_{1} & x_{2} & x_{3} & x_{4} \\
0 & 0 & 0 & 0
\end{array}\right)
$$

$X_{k}$ denotes the matrix obtained deleting from the matrix $X$ its $k$-th column and its last row. Then let $D_{k}=(-1)^{k+1} \operatorname{det}\left(X_{k}\right)$ for $k=1, \ldots, 4$.

The equations for the central configurations (1) of the 4-body problem were written by Dziobek [17] (see also equations (8) and (16) of Moeckel [35] or [24]) as the following 12 equations with 12 unknowns.

$$
\begin{aligned}
e_{i, j}=c_{1}+c_{2} \frac{D_{i} D_{j}}{m_{i} m_{j}}-\frac{1}{r_{i j}^{3}} & =0, \\
t_{i}-t_{j} & =0,
\end{aligned}
$$

for $1 \leq i<j \leq 4$, with

$$
t_{i}=\sum_{j=1, j \neq i}^{4} D_{j} r_{i j}^{2}
$$

The unknowns of equation (4) are the mutual distances $r_{i j}$, the variables $D_{i}$, and the constants $c_{k}(k=1,2)$.

The first six Dziobek's equation (4) are

$$
\begin{array}{ll}
m_{1} m_{2}\left(r_{12}^{-3}-c_{1}\right)=c_{2} D_{1} D_{2}, & m_{3} m_{4}\left(r_{34}^{-3}-c_{1}\right)=c_{2} D_{3} D_{4}, \\
m_{1} m_{3}\left(r_{13}^{-3}-c_{1}\right)=c_{2} D_{1} D_{3}, & m_{2} m_{4}\left(r_{24}^{-3}-c_{1}\right)=c_{2} D_{2} D_{4}, \\
m_{1} m_{4}\left(r_{14}^{-3}-c_{1}\right)=c_{2} D_{1} D_{4}, & m_{2} m_{3}\left(r_{23}^{-3}-c_{1}\right)=c_{2} D_{2} D_{3},
\end{array}
$$

where

$$
\begin{aligned}
& D_{1}=\left|\begin{array}{lll}
1 & c & b \\
0 & a & a \\
1 & 1 & 1
\end{array}\right|=a(c-b), \quad D_{2}=\left|\begin{array}{lll}
b & c & 0 \\
a & a & 0 \\
1 & 1 & 1
\end{array}\right|=a(b-c), \\
& D_{3}=\left|\begin{array}{lll}
0 & 1 & b \\
0 & 0 & a \\
1 & 1 & 1
\end{array}\right|=a, \quad D_{4}=\left|\begin{array}{ccc}
c & 1 & 0 \\
a & 0 & 0 \\
1 & 1 & 1
\end{array}\right|=-a .
\end{aligned}
$$

Multiplying equations (5) in order that each one at the right has the expression $c_{2}^{2} D_{1} D_{2} D_{3} D_{4}$, and since the masses must be positive we obtain the Dziobek relation:

$$
\left(r_{12}^{-3}-c_{1}\right)\left(r_{34}^{-3}-c_{1}\right)=\left(r_{13}^{-3}-c_{1}\right)\left(r_{24}^{-3}-c_{1}\right)=\left(r_{14}^{-3}-c_{1}\right)\left(r_{23}^{-3}-c_{1}\right) .
$$

The relation holds for every planar central configuration of the 4-body problem. 
We can solve $c_{1}$ from the Dziobek relation and we have

$$
\begin{aligned}
c_{1} & =\frac{r_{12}^{-3} r_{34}^{-3}-r_{13}^{-3} r_{24}^{-3}}{r_{12}^{-3}+r_{34}^{-3}-r_{13}^{-3}-r_{24}^{-3}} \\
& =\frac{r_{13}^{-3} r_{24}^{-3}-r_{14}^{-3} r_{23}^{-3}}{r_{13}^{-3}+r_{24}^{-3}-r_{14}^{-3}-r_{23}^{-3}} \\
& =\frac{r_{14}^{-3} r_{23}^{-3}-r_{12}^{-3} r_{34}^{-3}}{r_{14}^{-3}+r_{23}^{-3}-r_{12}^{-3}-r_{34}^{-3}} .
\end{aligned}
$$

Defining

$$
\begin{array}{ll}
s_{1}=r_{12}^{-3}+r_{34}^{-3}, & p_{1}=r_{12}^{-3} r_{34}^{-3}, \\
s_{2}=r_{13}^{-3}+r_{24}^{-3}, & p_{2}=r_{13}^{-3} r_{24}^{-3}, \\
s_{3}=r_{14}^{-3}+r_{23}^{-3}, & p_{3}=r_{14}^{-3} r_{23}^{-3},
\end{array}
$$

then equation (7) becomes

$$
c_{1}=\frac{p_{1}-p_{2}}{s_{1}-s_{2}}=\frac{p_{2}-p_{3}}{s_{2}-s_{3}}=\frac{p_{3}-p_{1}}{s_{3}-s_{1}}
$$

which imply that the point $\left(s_{1}, p_{1}\right),\left(s_{2}, p_{2}\right),\left(s_{3}, p_{3}\right)$ are on the same line with slope $c_{1}$, i.e.

$$
\left|\begin{array}{ccc}
1 & 1 & 1 \\
s_{1} & s_{2} & s_{3} \\
p_{1} & p_{2} & p_{3}
\end{array}\right|=0 .
$$

So we can write Dziobek equation as the following nice factorization

$$
D=\left(r_{13}^{3}-r_{12}^{3}\right)\left(r_{23}^{3}-r_{34}^{3}\right)\left(r_{24}^{3}-r_{14}^{3}\right)-\left(r_{12}^{3}-r_{14}^{3}\right)\left(r_{24}^{3}-r_{34}^{3}\right)\left(r_{13}^{3}-r_{23}^{3}\right)=0 .
$$

The equation $D=0$ must be satisfied for every planar central configuration of the 4-body problem.

\section{Proofs of Lemma 1 And Theorem 2}

For proving Lemma 1 we shall use Pitot's Theorem which states that in a tangential quadrilateral the two sums of lengths of opposite sides are the same, for a proof see for instance [27].

Proof of Lemma 1. By Pitot's and Pythagoras Theorems and Figure 1 we get that

$$
1+c-b=\sqrt{a^{2}+b^{2}}+\sqrt{(1-c)^{2}+a^{2}} .
$$

Isolating $c$ from this equality we obtain the conclusion of the lemma. 


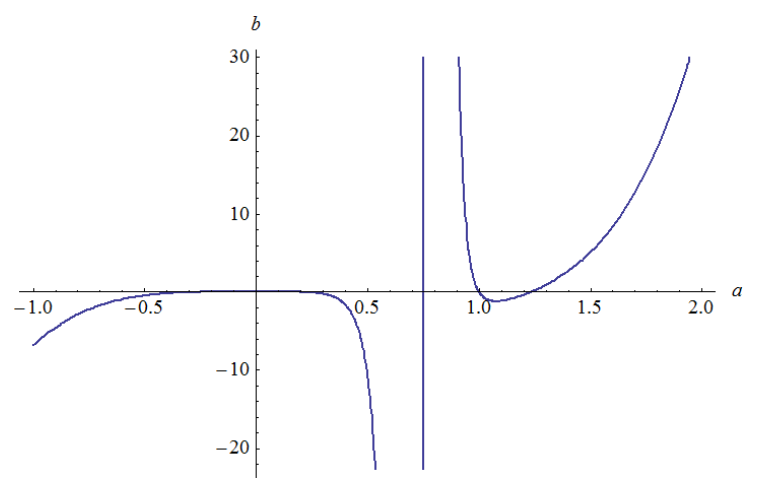

Figure 3. The graphic of Dziobek $=0$ for $a=1$.

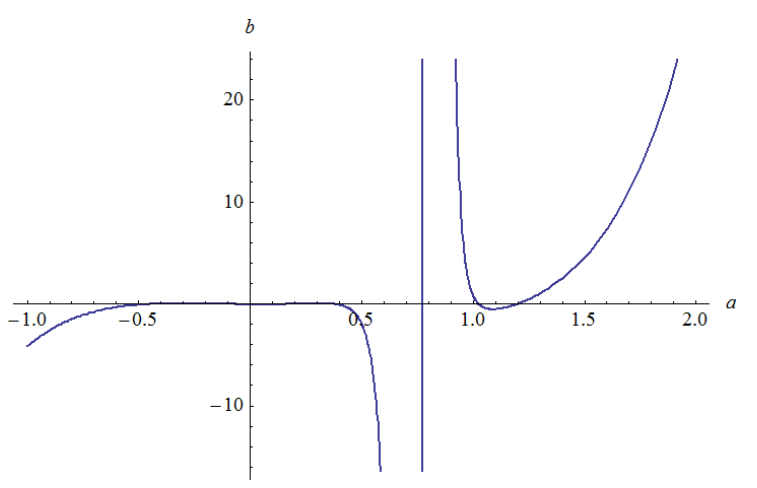

Figure 4. The graphic of Dziobek $=0$ for $a=0.95$.

Proof of Theorem 2. From (2) we have

(1)

$$
\begin{aligned}
& r_{12}=1, \quad r_{14}=\sqrt{a^{2}+b^{2}}, \quad r_{24}=\sqrt{a^{2}+(1-b)^{2}}, \\
& r_{13}=\sqrt{a^{2}+\frac{(1-b)^{2}\left(a^{2}+2\left(b+\sqrt{a^{2}+b^{2}}\right)\right)^{2}}{\left(a^{2}+4 b-4\right)^{2}}}, \\
& r_{23}=\sqrt{\frac{a^{2}+\frac{\left(a^{2}(b-2)+2(b-1)\left(b-2+\sqrt{a^{2}+b^{2}}\right)\right)^{2}}{\left(a^{2}+4 b-4\right)^{2}}}{\left(a^{2}+4 b-4\right)^{2}}} . \\
& r_{34}=\sqrt{\frac{\left(a^{2}-(b-1)\left(\sqrt{a^{2}+b^{2}}-b\right)\right)^{2}}{\left(a n^{2}\right.}} .
\end{aligned}
$$

Substituting these expressions together with the values of $D_{k}$ for $k=1,2,3,4$ into the last six equations of (4), we found that they are identically zero for a tangential trapezoid configuration. 


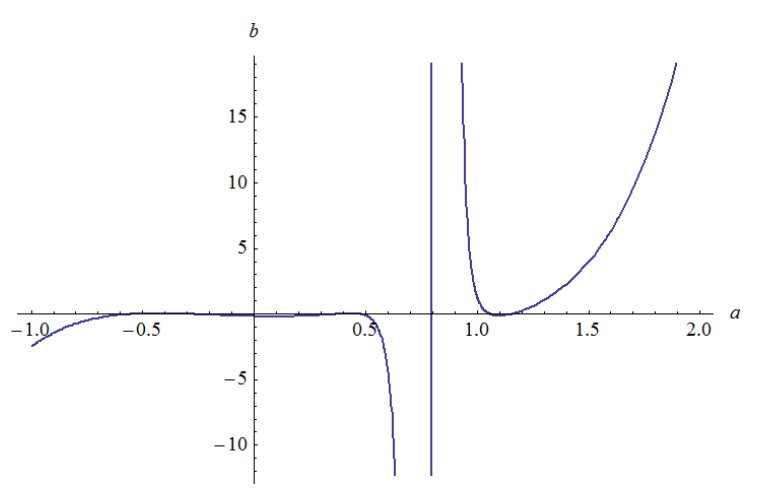

Figure 5. The graphic of Dziobek $=0$ for $a=0.9$.

Dividing conveniently two different equations of (5) we obtain

$$
\begin{aligned}
& \frac{m_{2}\left(r_{23}^{-3}-c_{1}\right)}{m_{1}\left(r_{13}^{-3}-c_{1}\right)}=\frac{D_{2}}{D_{1}}, \\
& \frac{m_{3}\left(r_{23}^{-3}-c_{1}\right)}{m_{1}\left(r_{12}^{-3}-c_{1}\right)}=\frac{D_{3}}{D_{1}}, \\
& \frac{m_{4}\left(r_{24}^{-3}-c_{1}\right)}{m_{1}\left(r_{12}^{-3}-c_{1}\right)}=\frac{D_{4}}{D_{1}} .
\end{aligned}
$$

Since $m_{1}=1$, using (7) and (11), we have

$$
\begin{aligned}
m_{2} & =\frac{D_{2} r_{23}^{3} r_{24}^{3}\left(r_{13}^{3}-r_{14}^{3}\right)}{D_{1} r_{13}^{3} r_{14}^{3}\left(r_{23}^{3}-r_{24}^{3}\right)}, \\
m_{3} & =\frac{D_{3} r_{23}^{3} r_{34}^{3}\left(r_{12}^{3}-r_{14}^{3}\right)}{D_{1} r_{12}^{3} r_{14}^{3}\left(r_{23}^{3}-r_{34}^{3}\right)}, \\
m_{4} & =\frac{D_{4} r_{24}^{3} r_{34}^{3}\left(r_{12}^{3}-r_{13}^{3}\right)}{D_{1} r_{12}^{3} r_{13}^{3}\left(r_{24}^{3}-r_{34}^{3}\right)} .
\end{aligned}
$$

Substituting these masses into the first six Dziobek equations (4), and taking only the numerators of these six equations because the denominators do not vanish, we have

$$
\begin{aligned}
e_{1,2}= & D_{2}\left(c_{2} D_{1}^{3} r_{12}^{3} r_{13}^{3} r_{14}^{2}\left(r_{24}^{3}-r_{23}^{3}\right)-r_{23}^{3} r_{24}^{3}\left(c_{1} r_{12}^{3}-1\right)\left(r_{13}^{3}-r_{14}^{3}\right)\right), \\
e_{1,3}= & D_{3}\left(c_{2} D_{1}^{2} r_{12}^{3} r_{13}^{3} r_{14}^{3}\left(r_{34}^{3}-r_{23}^{3}\right)-r_{23}^{3} r_{34}^{3}\left(c_{1} r_{13}^{3}-1\right)\left(r_{12}^{3}-r_{14}^{3}\right)\right), \\
e_{2,3}= & D_{2} D_{3}\left(r_{23}^{3} r_{24}^{3} r_{34}^{3}\left(c_{1} r_{23}^{3}-1\right)\left(r_{12}^{3} r_{14}^{3}\right)\left(r_{14}^{3}-r_{13}^{3}\right)-\right. \\
& \left.c_{2} D_{1}^{2} r_{12}^{3} r_{13}^{3} r_{14}^{6}\left(r_{23}^{3}-r_{24}^{3}\right)\left(r_{23}^{3}-r_{34}^{3}\right)\right), \\
e_{1,4}= & D_{4}\left(c_{2} D_{1}^{2} r_{12}^{3} r_{13}^{3} r_{14}^{3}\left(r_{34}^{3}\right)-r_{24}^{3}-r_{24}^{3} r_{34}^{3}\left(c_{1} r_{14}^{3}-1\right)\left(r_{12}^{3}-r_{13}^{3}\right)\right), \\
e_{2,4}= & D_{2} D_{4}\left(-r_{23}^{3} r_{24}^{3} r_{34}^{3}\left(c_{1} r_{24}^{3}-1\right)\left(r_{12}^{3}-r_{13}^{3}\right)\left(r_{13}^{3}-r_{14}^{3}\right)-\right. \\
& \left.c_{2} D_{1}^{2} r_{12}^{3} r_{13}^{6} r_{14}^{3}\left(r_{23}^{3}-r_{24}^{3}\right)\left(r_{24}^{3}-r_{34}^{3}\right)\right), \\
e_{3,4}= & D_{3} D_{4}\left(c_{2} D_{1}^{2} r_{12}^{6} r_{13}^{3} r_{14}^{3}\left(r_{23}^{3}-r_{34}^{3}\right)\left(r_{34}^{3}-r_{24}^{3}\right)-\right. \\
& \left.r_{23}^{3} r_{24}^{3} r_{34}^{3}\left(c_{1} r_{34}^{3}-1\right)\left(r_{12}^{3}-r_{13}^{3}\right)\left(r_{12}^{3}-r_{14}^{3}\right)\right) .
\end{aligned}
$$


Notice that $D_{i}(i=1,2,3,4)$ is non-zero, so we can eliminate $D_{i}$ from equations (13). First, we solve the first two equations with respect to $c_{1}$ and $c_{2}$, and then we substituted $c_{1}$ and $c_{2}$ in the last four equations of (13). We obtain

$$
\begin{aligned}
& e_{2,3}=\frac{D}{d} r_{23}^{6} r_{24}^{3} r_{34}^{3}\left(r_{14}^{3}-r_{12}^{3}\right)\left(r_{14}^{3}-r_{13}^{3}\right), \\
& e_{1,4}=0, \\
& e_{2,4}=\frac{D}{d} r_{23}^{3} r_{24}^{6} r_{34}^{3}\left(r_{13}^{3}-r_{12}^{3}\right)\left(r_{13}^{3}-r_{14}^{3}\right), \\
& e_{3,4}=\frac{D}{d} r_{23}^{3} r_{24}^{3} r_{34}^{6}\left(r_{13}^{3}-r_{12}^{3}\right)\left(r_{12}^{3}-r_{14}^{3}\right),
\end{aligned}
$$

where $D=0$ is the Dziobek equation (9), and

$$
d=r_{12}^{3}\left(r_{13}^{3} r_{23}^{3}\left(r_{24}^{3}-r_{34}^{3}\right)+r_{14}^{3} r_{24}^{3}\left(r_{34}^{3}-r_{23}^{3}\right)\right)+r_{13}^{3} r_{14}^{3} r_{34}^{3}\left(r_{23}^{3}-r_{24}^{3}\right),
$$

is the denominator which comes from the denominator of $c_{1}$ and $c_{2}$.

In conclusion the tangential trapezoid central configurations must satisfy $\left(e_{2,3}, e_{2,4}, e_{3,4}\right)=$ $(0,0,0)$.

Substituting (10) into $e_{2,3}=0, e_{2,4}=0, e_{3,4}=0$, we get that these three equations are satisfied if and only if the following equations

$$
\begin{aligned}
& E_{2,3}=D W_{1} W_{3}=0, \\
& E_{2,4}=D W_{2} W_{3}=0, \\
& E_{3,4}=D W_{1} W_{2}=0,
\end{aligned}
$$

have solutions respectively, where

$$
\begin{aligned}
& W_{1}=1-\sqrt{a^{2}+b^{2}}, \\
& W_{2}=1-\sqrt{a^{2}+\frac{(b-1)^{2}\left(a^{2}+2\left(b+\sqrt{a^{2}+b^{2}}\right)\right)^{2}}{\left(a^{2}+4 b-4\right)^{2}}} \\
& W_{3}=\sqrt{a^{2}+b^{2}}-\sqrt{a^{2}+\frac{(b-1)^{2}\left(a^{2}+2\left(b+\sqrt{a^{2}+b^{2}}\right)\right)^{2}}{\left(a^{2}+4 b-4\right)^{2}}},
\end{aligned}
$$

and $D=0$ is the Dziobek equation (9).

The solutions of $\left\{E_{2,3}, E_{2,4}, E_{3,4}\right\}=0$ and of Dziobek $=0$ having positive masses are the tangential trapezoid central configurations of the four-body problem.

First we solve equations $\left\{E_{2,3}, E_{2,4}, E_{3,4}\right\}=0$. Using Mathematica, we obtain that

$$
a=\frac{\sqrt{3}}{2}, \quad b=-\frac{1}{2}, \quad m_{2}=1.5124659 . ., \quad m_{3}=1, \quad m_{4}=1.5124659 \ldots
$$

Next we find solutions of Dziobek $=0$. In order to solve Dziobek $=0$, we use Mathematica. We plot the graphic of Dziobek $=0$ when $a=1,0.95,0.9$, see Figures 3, 4 , and 5 .

When $a=1$ we solve Dziobek $=0$ and get $b=0$, and from (12) we obtain $m_{1}=m_{2}=m_{3}=m_{4}=1$. 
When $a=0.95$ we solve Dziobek $=0$ and get the three solutions

$$
b=-0.3122499 . ., \quad b=0.3122499 . ., \quad b=1.02234 \ldots
$$

Then from (12) we have

$$
\begin{array}{lll}
m_{2}=0.49701 . ., & m_{3}=1, & m_{4}=0.49701 . ., \quad \text { for }(a=0.95, b=-0.31224 . .), \\
m_{2}=2.01201 . ., & m_{3}=1, & m_{4}=2.01201 . ., \quad \text { for }(a=0.95, b=0.31224 . .), \\
m_{2}=1.09839 . ., & m_{3}=0.7557 . ., & m_{4}=0.63245 . ., \quad \text { for }(a=0.95, b=1.02234 . .) .
\end{array}
$$

When $a=0.9$ we solve Dziobek $=0$ and get the three solutions

$$
b=-0.4358899 . ., \quad b=0.4358899 . ., \quad b=1.05179 \ldots
$$

Therefore from (12) we have

$$
\begin{array}{llll}
m_{2}=0.20834 . ., & m_{3}=1, & m_{4}=0.20834 . ., & \text { for }(a=0.9, b=-0.43588 . .), \\
m_{2}=4.79976 . ., & m_{3}=1, & m_{4}=4.79976 . ., & \text { for }(a=0.9, b=0.43588 . .), \\
m_{2}=1.14928 . ., & m_{3}=0.51298 . ., & m_{4}=0.27025 . ., & \text { for }(a=0.9, b=1.05179 . .) .
\end{array}
$$

When $a$ goes from 1 to $\frac{\sqrt{3}}{2}$, the solutions for Dziobek $=0$ has two symmetric solutions in the interval $\left(-\frac{1}{2}, \frac{1}{2}\right)$ and one solution in the interval $\left(1, \frac{11}{10}\right)$ with positive masses, and we have

$$
\begin{gathered}
m_{1}=m_{3}=1, m_{2}=m_{4}>1 \quad \text { for } \quad 0 \leq b<\frac{1}{2}, \\
m_{1}=m_{3}=1, m_{2}=m_{4}<1 \quad \text { for } \quad-\frac{1}{2}<b \leq 0, \\
m_{2}>m_{1}=1>m_{3}>m_{4} \quad \text { for } \quad 1 \leq b<\frac{11}{10}
\end{gathered}
$$

We find that $m_{2}=m_{4}$ as $(a, b) \rightarrow\left(\frac{\sqrt{3}}{2}, \frac{1}{2}\right)$, and $m_{2}=m_{4} \rightarrow 0$ as $(a, b) \rightarrow$ $\left(\frac{\sqrt{3}}{2},-\frac{1}{2}\right)$.

In summary studying the graphic of $D=0$ with a varying from 1 to $\frac{\sqrt{3}}{2}$, we obtain the statements of Theorem 2 .

\section{ACKNOWLEDGEMENTS}

We thank to the reviewer his/her detailed report which help us to improve the presentation of our results.

The first author is partially supported by the Ministerio de Economía, Industria y Competividad, Agencia Estatal de Investigación grant MTM 2016-77278-P (FEDER), the Agència de Gestió d'Ajuts Universitaris i de Recerca grant 2017 SGR 1617, and the H2020 European Research Council grant MSCA-RISE-2017-777911. The second author is partially supported by Fundamental Research Funds for the Central Universities (NO.XDJK2015C139), China Scholarship Council (Number 201708505030). 


\section{REFERENCES}

[1] Albouy, A., Symétrie des configurations centrales de quatre corps, C. R. Acad. Sci. Paris, 320 (1995), 217-220.

[2] Albouy, A. Recherches sur le problème des n corps, Notes Scientifiques et Techniques du Bureau des Longitudes, Pairs, 1997, pp. 78.

[3] Albouy, A., Chenciner, A., Le problème des n corps et les distances mutuelles, Invent. Math. 131 (1998), 151-184.

[4] Albouy, A. and Fu, Y., Euler configurations and quasi polynomial systems, Regul. Chaotic Dyn. 12 (2007), 39-55.

[5] Albouy, A., Fu, Y. and Sun, S., Symmetry of planar four body convex central configurations, Proc. R. Soc. Lond. Ser. A Math. Phys. Eng. Sci., 464 (2008), 1355-1365.

[6] Albouy, A. and Kaloshin, V., Finiteness of central configurations of five bodies in the plane, Ann. of Math. (2) 176 (2012), 535-588.

[7] Álvarez, M. and Llibre, J., The symmetric central configurations of the 4-body problem with masses $m_{1}=m_{2} \neq m_{3}=m_{4}$, Appl. Math. Comp. 219 (2013), 5996-6001.

[8] Álvarez, M. and Llibre, J., Hjelmslev quadrilateral central configurations, Physics Letters A 383 (2018), 103-109.

[9] Álvarez, M. and Llibre, J., Equilic quadrilateral central configurations, Commun. Nonlinear Sci. Numer. Simul. 78 (2019), 104872, 7 pp.

[10] Alvarez-Ramirez, A., Santos, A.A., and Vidal, C., On co-circular central configurations in the four and five body-problem for homogeneous force law, J.Dynam. Differential Equations 25 (2013), no.2, 269-290.

[11] Arenstorf, R.F., Central configurations of four bodies with one inferior mass, Cel. Mechanics 28 (1982), 9-15.

[12] Barros, J.F. and Leandro, E.S.G., The set of degenerate cetral configurations in the planar restricted four-body problem, SIAM Journal on Mathematical Analysis 43 (2011), 634-661.

[13] Barros, J.F. and Leandro, E.S.G., Bifurcations and enumeration of classes of relative equilibria in the planar restricted four-body problem, SIAM Joural on Mathematical Analysis 46 (2014), 1185-1203.

[14] Bernat, J., Llibre, J. and Perez Chavela, E., On the planar central configurations of the 4-body problem with three equal masses, Dyn. Contin. Discrete Impuls. Syst. Ser. A Math. Anal. 16 (2009), 1-13.

[15] Corbera, M. and Llibre, J., Central configurations of the 4-body problem with masses $m_{1}=$ $m_{2}>m_{3}=m_{4}=m>0$ and $m$ small, Appl. Math. Comput. 246 (2014), 121-147.

[16] Corbera, M., Cors, J.M.,Llibre, J. and Perez-Chavela, E., Trapezoid central configurations, Appl. Math. and Comput. 346 (2019), 127-142.

[17] Cors J.M. and Roberts G.E., Four-body co-circular central configurations, Nonlinearity 25 (2012), 343-370.

[18] Davis, C., Geyer, S., Johnson, W. and Xie, Z., Inverse problem of central configurations in the collinear 5-body problem, J. Math. Phys. 59 (2018), no. 5, 052902, 18 pp.

[19] Dziobek, O., Ueber einen merkwürdigen Fall des Vielkörperproblems, Astro. Nach. 152 (1900), 32-46.

[20] Érdi, B. and Czirják, Z., Central configuration of four bodies with an axis of symmetry, Celestial Mech.Dynam. Astronom. 125 (2016), no.1, 33-70.

[21] Euler, L., De moto rectilineo trium corporum se mutuo attahentium, Novi Comm. Acad. Sci. Imp. Petrop., 11 (1767), 144-151.

[22] Fernandes, A.C., Garcia, B.A., Llibre, J. and Mello, L.F., New central configurations of the $(\mathrm{n}+1)$-body problem, J. Geom. Phys. 124 (2018), 199-207.

[23] Fernandes, A.C., Llibre, J. and Mello, L.F., Convex central configurations of the 4-body problem with two pairs of equal masses, Arch. Rational Mech. Anal. 226 (2017), 303-320.

[24] Gannaway, J.R., Determination of all central configurations in the planar 4-body problem with one inferior mass, Ph. D., Vanderbilt University, Nashville, USA, 1981.

[25] Hagihara, Y., Celestial Mechanics, vol. 1, MIT Press, Massachusetts, 1970.

[26] Hampton, M. and Moeckel, R., Finiteness of relative equilibria of the four-body problem, Invent. Math. 163 (2006), no.2, 289-312.

[27] Josefson, M., More characterizations of tangential quadrilaterals, Forum Geometricorum 11 (2011), 65-82. 
[28] Largange, J.L., Essai surle probléme des trois corps, recueil des pièces qui ont remporté le prix de l'Académie royale des Sciences de Paris,tome IX,1772, reprinted in Ouvres, Vol.6(GauthierVillars,Paris,1873), pp 229-324.

[29] Leandro, E.S.G., Finiteness and bifurcation of some symmetrical classes of central configurations, Arch. Rational Mech. Anal. 167 (2003), 147-177.

[30] Llibre, J., Posiciones de equilibrio relativo del problema de 4 cuerpos, Publica cions Matemàtiques UAB3 (1976), 73-88.

[31] Llibre, J., On the number of central configurations in the N-body problem, Celestial Mech. Dynam. Astronom. 50 (1991), 89-96.

[32] Long, Y., Admissible shapes of 4-body non-collinear relative equilibria, Adv. Nonlinear Stud. 3 (2003), no. 4, 495-509.

[33] Long, Y. and Sun, S., Four-Body Central Configurations with some Equal Masses, Arch. Rational Mech. Anal. 162 (2002), 24-44.

[34] MacMillan, W.D. and Bartky, W., Permanent Configurations in the Problem of Four Bodies, Trans. Amer. Math. Soc. 34 (1932), no. 4, 838-875.

[35] Moeckel, R., On central configurations, Mathematische Zeitschrift 205 (1990), no. 4, $499-517$.

[36] Moeckel, R., Generic finiteness for Dziobek configurations, Trans. Amer. Math. Soc. 353 (2001), 4673-4686.

[37] Meyer, K.R., Bifurcation of a central configuration, Cel.Mech. 40 (1987), 273-282.

[38] Moulton, F.R., The straight line solutions of n bodies, Ann. of Math. 12 (1910), 1-17.

[39] Palmore, J.I., Classifying relative equilibria II, Bull. Amer. Math. Soc. 81(1975), 71-73.

[40] Pedersen, P., Librationspunkte im restringierten Vierkörperproblem, Danske Vid. Selsk. Math.Fys. 21 (1944), 1-80.

[41] Pérez-Chavela E., Santoprete, M., Convex four-body central configurations with some equal masses, Arch. Rational Mech. Anal. 185 (2007), 481-494.

[42] Piña, E., Lonngi, P., Central configuration for the planar Newtonian four-body problem, Celest. Mech. Dyn. Astron. 108 (2010), 73-93.

[43] Saari, D.G., On the role and properties of central configurations, Celestial Mech., 21 (1980), 9-20.

[44] Schmidt, D.S., Central configurations in $\mathbb{R}^{2}$ and $\mathbb{R}^{3}$, Contemporary Math. 81(1980), 59-76.

[45] Simó, C., Relative equilibrium solutions in the four-body problem, Cel. Mechanics 18 (1978), 165-184.

[46] Smale, S., Topology and mechanics II. The planar n-body problem, Invent.Math. 11 (1970), 45-64.

[47] Wintner, A., The Analytical Foundations of Celestial Mechanics, Princeton Math. Series 5, Princeton University Press, Princeton, NJ, 1941.

[48] Xia, Z., Central configurations with many small masses, J. Differential Equations 91 (1991), 168-179.

[49] Xia, Z., Convex central configurations for the n-body problem, J. Differential Equations 200 (2004), 185-190.

[50] Xie, Z., Isosceles trapezoid central configurations of the Newtonian four-body problem, Proc. R. Soc. Edinb., Sect. A, Math. 142 (2012), 665-672.

1 Departament de Matemàtiques, Universitat Autònoma de Barcelona, 08193 Bellaterra, Barcelona, Catalonia, Spain

Email address: jllibre@mat.uab.cat

2 School of Mathematics and Statistics, Southwest University, 400715, Chongqing, CHINA

Email address: yuanpengfei@swu.edu.cn 